作 Denmark; ${ }^{3}$ Department of Business Development and Technology, Aarhus University, Denmark; ${ }^{4}$ Interdisciplinary Centre for Organizational Architecture, Department of 9 Management, Aarhus University, Denmark; ${ }^{5}$ Department of Bioscience, Aarhus University, Denmark

Collaborative decision making is central to the organization of society. Juries deliberate cases, voters elect government officials, open innovation networks converge on innovative solutions. It is common to think of such groups as decision making entities. But this language is imprecise. Real decision processes do not occur within any group or organization as an abstract entity. Collaborative decision making happens within and between autonomous individuals. This emphasizes the importance of the relationships between individual and social decision-making processes to social organization. Despite a rich body of literature on collaborative decision making, we know little about how individuals decide to commit to decision making groups in the first place; and how, once joined, they communicate their distributed information for optimal group performance. Using a synthesis of cognitive models and agent-based simulation, we investigate the influence of information flow on commitment and performance in open collaborative decision making groups. The main results of the simulations are that 1) commitment is enhanced when groups are designed so agents have realistic knowledge about the forgone gains and losses associated with choosing to join a decision making group; and 2) that collaborative decision making groups gain most when communication between members conveys more information about individual's preferences. We thus demonstrate that collaborative decision making is done best when it is done by groups that are informationally open.

A property of agents in real collaborative decision-making groups is autonomy. This has an important consequence that has been largely bracketed off in most research on collaborative decision-making (an important exception in artificial intelligence is (1)). Real agents are free to do more than decide between alternatives in the context of some group; they are also free to decide whether or not they will participate in the group at all. This is true in unconstrained contexts, like politics (e.g. $(2,3))$ as well as is in highly constrained contexts, like juries. Not only are voters and jurors free to decide their own vote, they are at least practically free to abstain from voting at all.

This raises questions about how individuals in groups evaluate, communicate, and decide; not only about choice alternatives, but also about the groups of which they are members.

Such questions are increasing in social importance. This is visible in the host of seemingly more 'open' organizational forms and crowd type phenomena including crowdsourcing and user innovation (4-8), which require individuals to have greater second-order autonomy of the kind described. These forms abstain from the notion of a sole managerial decision maker handling decisions about what activities should be initiated. Instead, they deliberately aim to harness and aggregate the unique knowledge and ideas of each individual member, and to essentially serve as a mechanism for pooling the knowledge of individual actors via collective action.

With no managerial intervention, individuals in these forms and crowds are not required to engage in any given team. This means that smaller decision making units within the larger form are frequently formed and disbanded (e.g. (8,9)). Agents are autonomous, and only likely to engage in collaborative efforts if these are perceived valuable to the individual agent. The simulations we present here are designed specifically to address the autonomy of agents within such structurally open groups

The conceptual focus of our model is on information; or more fully, on how variations in the representation and communication of choice information may influence autonomous agents' commitment to, and performance in, collaborative decision-making groups. We identify these processes as particularly important, because they are relatively easily influenced by founders and coordinators of decentralized groups. As such, these processes may serve as points of control for optimizing commitment and collective gain.

The first is the process of judging the value of joining a collaborative decision making group, compared to the value of abstaining and making one's decisions alone. Such judgment is complicated, because it involves calibrating one's own preferences against outcomes arising from the collaboratively made decision. This calibration is complex, because it depends on the knowledge one has about gains and losses that are forgone in the decision to collaborate. The problem the agent faces in this context, and the role of knowledge of forgone personal outcomes in solving this problem, is best illustrated by an example.

Imagine that you are a member of a property investment group. Your own preference is to invest in residential property, but the group's overall choice is to invest in retail property. In deciding to join the group, you commit to its decision, and so forgo acting on you own preference. The question this raises is the following: if the retail investment yields a large gain, how will this outcome influence your evaluation of the group and your tendency to work with it in the future?

The simplest intuition is that your evaluation is purely a function of the return on your investment. If the return is large, then you will value the group more highly, irrespective of the conflict between your preference and the group choice. There is an alternative intuition, which is more complex, and perhaps more plausible. Consider the case in which the retail investment yields a large gain, yet underperforms the average

${ }^{2}$ To whom correspondence should be addressed. E-mail: filjcs@cas.au.dk All code is made available on the Open Science Framework (https://osf.io/t7xbz/) 
residential investment. In this case, your personal preferences will likely interact in how you update your evaluation of the group. Research on knowledge of forgone outcomes (10-12); particularly research on the role of regret in decision-making $(13,14)$; indicates that decision-makers in these contexts may devalue the group, even if the received payoff from committing to the group is large. In other words, if knowledge of individual and group outcomes is symmetrical, then a comparison between the two is likely to affect evaluation of the group.

The crucial question then is: what happens if you don't have this knowledge? What happens if knowledge of group and individual outcomes is asymmetrical? One possibility is that in the absence of knowledge you will default to the simple case, and evaluate the group as a pure function of gain. A more likely possibility is that expectations will fill in to play the role of knowledge(14). In other words, in the absence of knowledge, you will update your evaluation of the group by comparing the outcome you did receive, to what you believe you would have received, had the group chosen your preferred option. If you had a very strong preference for a residential investment, and the group voted for a retail investment, then unless the chosen property outperforms your high expectations for your preferred choice, you may be likely to devalue the collaboration, even if the group choice yielded a gain. The assumption here is that in lieu of counterfactual knowledge of forgone outcomes, agents will rely on imagined outcomes to update their expectancies.

Although the example is schematized, it highlights important features of all kinds of open, iterative, and contractual decision-making groups in which people freely participate. More natural examples include investment groups generally; home owner associations; funding agencies; and hiring committees. We incorporate this key difference in our model, and investigate the influence of this kind of counterfactual knowledge of outcomes on commitment and decision performance in the simulations below.

A separate important informational process in collaborative decision-making is communication between agents. Communication in collaborative decision making can vary in depth and complexity, from the casting of ballots in elections, to the extended verbal deliberations of juries. The methods used for communicating and aggregating information within groups have important consequences for group performance (8). Given that most important decisions are uncertain, one method of information aggregation which is particularly important is the sharing and calibration of confidence between group members(15). Confidence sharing has been shown to improve the accuracy of judgments made by dyads $(16,17)$; to introduce sub-optimalities from pro-social biases into judgment (18); to influence collective learning (19); and to introduce systematic errors in group judgment $(18,20)$. This research shows that confidence sharing is a complex issue; at times facilitating and at times inhibiting group decisions. Thus we compare commitment and performance between cases in which confidence sharing is possible, and cases in which groups make a blind vote without direct communication of confidence, in the simulations below.

Both processes - judging the value of joining a decision making group, and communicating decisions within a group once joined - are likely to be shaped by the uncertainty of the context in which the decision is made. Research suggests that autonomously chosen groups - like the group in the property 187 investment example - reliably outperform individual forecast- 188 ers and other kinds of groups when making risky judgments. 189 A prominent example is provided by prediction or information 190 markets (21). In prediction markets, individuals autonomously 191 trade contracts on the outcome of future uncertain events - 192 for instance elections (22), geopolitical events (23), or climate 193 events (24)- to be paid out after the event occurs. The mar- 194 ket price for each outcome is interpretable as the group's 195 aggregated judgment of its likelihood. Interpreted this way, 196 prediction markets have been shown to provide more accurate 197 forecasts than other forms of aggregated judgment, such as 198 exit polls in elections. It is possible that prediction markets 199 gain this advantage by affording mechanisms for learning to 200 calibrate risk and uncertainty against decision utility at the 201 group level (23), however this remains an open research ques- 202 tion (see the chapters in(21) for detailed discussions). The 203 simulations reported here are also designed to contribute to 204 this debate.

\section{Details of the Model}

208

To formulate the above questions in a more precise, theoreti- 209 cally informed way, we employ large-n cognitive agent-based 210 simulation. This method allows us to use simple and well 211 understood cognitive models of individual behavior, to de- 212 duce emergent properties of decision-making in decentralized 213 groups, with individual agents interacting under sets of con- 214 ditions which are of direct relevance to the above concerns. 215 This approach combines the strengths of cognitive modeling; 216 including clear, conceptually well defined, and empirically 217 well-verified mathematical explanations of cognitive processes 218 causing human choice behavior, with the strengths of agent 219 based modeling; including a focus on the effects of structural 220 and environmental causes on emergent interactive properties 221 of group choice. Thus the method affords a synthesis of inter- 222 nal, microlevel explanations of individual choice with external, 223 macrolevel explanation of group choice. The code for the 224 following simulations is freely available on the Open Science 225 Framework (https://osf.io/t7xbz/).

To capture the autonomous nature of agents in decen- 227 tralized groups, we model collaborative decision-making as 228 a simple, two-step process. Agents first decide whether to 229 join a group or not, and only then do they choose between 230 alternatives in their task environment. We model the group 231 choice as a binary commitment. If agents decide to join the 232 group, their subsequent choice of alternatives is necessarily 233 aggregated with other group members, and a group decision 234 is made. Group members always receive the outcome of the 235 group decision, even if it conflicts with their individual choice. 236 If agents decide to abstain from the group, then their choice is 237 made alone, and they always receive their individually chosen 238 outcome.

To model decision-making as a process of learning how 240 to calibrate utility against risk and uncertainty, we model 241 decisions in a task environment designed with this purpose 242 in mind. The environment we choose for this purpose is the 243 Iowa Gambling Task (IGT: $(25,26))$.

The IGT is a biased multi-armed bandit game. Players are 245 asked to repeatedly choose cards from one of four available 246 decks, labelled A, B, C, and D. Each card represents either a 247 payout or a loss. The IGT is biased because payouts and losses 248 
249 are distributed asymmetrically across the four decks. Payouts 250 are distributed so that decks A and B seem immediately more 251 attractive - decks A and B have higher payouts (100 units) 252 than the decks C and D (50 units). The catch is that decks A 253 and $\mathrm{B}$ also include much heavier average losses (-1250 units 254 per 10 cards) than decks B and C (-250 units per 10 cards). 255 The result is that despite their more attractive immediate 256 gains, decks A and B (i.e. the "Bad" decks) lead to much 257 greater losses in the long run than decks C and D (i.e. the 258 "Good" decks).

The IGT is also biased because risk is distributed asym261 metrically across the decks. The game is designed so that the asymmetry in risk interacts with the asymmetry in average utility. For each of the Bad and the Good sets of decks, one deck is more transparently risky than the other. Deck A contains frequent but smaller losses, with a loss of -250 drawn every other card (on average). The result is that selection of deck A leads to long term loss, but risk is readily learned by the player. Deck B also contains lower mean utility, but risk is much less transparent. Deck B contains infrequent but much larger losses, with a loss of -1250 drawn only every 10 cards. Selection of deck B thus leads to the same long term loss as selection of deck A. However, because the risk is less transparent, it is not as readily learned. Similarly, in the Good decks, deck $\mathrm{C}$ contains higher utility with more transparent risk, with losses of -50 drawn every other card, and deck D contains higher utility with less transparent risk, with losses of -250 every 10 cards. It is this payoff structure that makes the IGT an appropriate context to investigate calibration of utility and risk in decentralized decision-making. (For discussion of use of multi-armed bandit tasks in organizational agent-based modeling contexts more generally, see $(8,27))$.

We model individual agents' learning and choice using the Prospect Valence Learning Delta (PVL-Delta) model (28-31). A wide range of cognitive models have been proposed to explain choice behavior on the IGT. These include strategy switching, Bayesian updating, and reinforcement learning families of models. Reinforcement learning models have proven the most appropriate, yielding improved information criterion scores over other kinds of models (31).

Within the class of reinforcement learning models, the PVLDelta model has been shown to generate performance which most closely resembles real human behavior, and the model has consistently performed best in direct model comparison (28-30). Thus the PVL-Delta model provides the most realistic model of human performance on the IGT currently available.

This is important, because it adds a high degree of cognitive realism to our simulations. By embedding a well validated, empirically confirmed cognitive model of the IGT within our agent-based approach, we have good reason to suppose that the emergent properties of group behavior which we observe in our simulations should also occur in groups of human agents.

The PVL-Delta model assumes agents solve the IGT using three interacting computational processes. The first is the transformation of the value received (or lost) into subjective utility following Prospect Theory (32). This process captures behavioral phenomena observed in human evaluation; including sensitivity to change rather than absolute value, loss-aversion, and decreasing subjective value associated with increasing objective returns and losses. Objective outcomes are transformed using the Prospect Utility function.

$$
u_{k}(t)= \begin{cases}X(t)^{A} & X \geq \rho \\ -w \times|X(t)|^{A} & X<\rho\end{cases}
$$

The function represents the subjective utility $u$ of the outcome received from deck $k$ (where $k$ may be $\mathrm{A}, \mathrm{B}, \mathrm{C}$, or $\mathrm{D}$ ), following selection on trial $t$. The measurement on a trial $X(t)$ represents the objective reward or loss received on the trial The function has a reference point $\rho$, which is the objective value above which all outcomes are experienced as a reward, and below which all outcomes are experienced as a loss. The present implementation assumes $\rho=0$ for all simulations. The parameter $A$ represents the concavity/convexity of the prospect utility function, designed to capture the decreasing influence that increasing losses and rewards have on decision maker's subjective evaluation, and thus agent's risk preferences for losses and gains. The parameter $w$ represents the asymmetry in the utility function between losses and rewards a feature of Prospect Theory which is designed to capture the tendency of decision makers to allow losses to have a greater influence on their evaluation.

The second process is learning of associations between choice alternatives and their outcomes. The model stores these associations as representations of expected value for each deck (i.e. value expectancies). These representations are updated on the basis of Prospect Theory transformed gains and losses, weighted according to a delta learning rule.

$$
E u_{k}(t)=E u_{k}(t-1)+a \times\left(u_{k}(t)-E u_{k}(t-1)\right)
$$

Here, the expected value $E_{u} k$ for deck $k$ following selection on trial $t$ is updated as a combination of the already stored expected utility for the chosen deck, and the subjective utility of the outcome of the previous choice. Importantly, the relative influence of these two factors - i.e. evaluations of each deck prior to receiving a choice outcome, and the subjective utility of the outcome received - are modulated by the learning rate parameter $a$. A higher value for this parameter means that the most recent outcome will be more highly weighted, and that the agent will more quickly "forget" losses and payoffs from older trials (see (8) for discussion of use of such rules in modeling collaborative decisions in organizational contexts).

The third process is the use of expectancies to inform choice. This is implemented using a softmax choice rule.

$$
P x_{k}(t+1)=\frac{e^{\theta \times E u_{k}(t)}}{\sum_{j=1}^{4} e^{\theta \times E u_{j}(t)}}
$$

The choice rule converts the agent's representation of relative expected value into relative probabilities for choosing each outcome, with more highly evaluated decks being more likely to be chosen on the next trial. The rule incorporates a sensitivity parameter $\theta$, which is a re-parameterization of a response consistency parameter $c$

$$
\theta=2^{c}-1
$$

which determines agents' tendency to exploit expected values in making their choice. Agents with a lower consistency parameter will tend more to explore choice options which have a lower expected value (8). 
By building on these three processes, we start from a model of individual learning and decision which is empirically validated at the individual level (29); behaviouraly plausible at the group level (8); and possible to implement in laboratory settings $(25,26)$.

We extend the PVL-Delta model to a collaborative decisionmaking context by generating a set of PVL-Delta agents, who all choose from the same deck of cards. To model structural openness, all agents are free to choose whether or not to decide collaboratively prior to each trial. Importantly, we assume that both decision steps - the decision to join the group, and the decision about which card to choose - implement the same underlying computational processes, implemented in the PVL-Delta model.

This assumption is parsimonious. It allows us to use the same core set of computational processes to model agent learning about the value of choice alternatives, and about the value of the group itself. Importantly, this assumption is also empirically justifiable. There is more to social decision-making than considerations of prospective value and uncertainty. Indeed, research on civic group affiliation shows that people do decide to join and leave groups for reasons that are independent of risk and utility considerations. Such reasons include personal affiliation and attraction (33) and social identity (34). However, reasons of risk, gain, and cost - such as the estimated probability that the group will meet its goals; the time taken to participate; and costs in terms of dues and fees - have been shown to be more important than these other factors (34). Thus each agent implements two separate PVL-Delta models the basic model, and a higher order model which implements the decision whether or not to commit to the collaboration on each trial.

Modeling the collaboration requires a small change in the basic PVL-Delta model. If an agent commits to the collaboration on a trial, then its own choice of deck on that trial cannot determine the outcome the agent receives. Rather, all collaborating agents pool their choices in a vote, and the deck with the most votes is represented as the choice for all individuals within the group. This means that for collaborating agents, expected value $E_{u} k$ for deck $k$ represents the outcome of the group choice, or winning vote, and not the agent's own choice. For agents who opt to choose their card independently, $E_{u} k$ updates normally.

From here, our model addresses the questions raised in the introduction as follows. When modeling the decision to collaborate, we assume that agents' expected values for committing to the group, versus deciding alone, are updated not on the basis of choice outcomes, but rather on the basis of a comparison between individual and collaborative rewards and losses. Specifically, we assume that agents' evaluation of the decision to collaborate increases whenever it perceives that the group decision leads to a greater outcome than its own choice of deck, and decreases whenever it perceives that the group outcome is worse than its own individual choice.

The implementation for this assumption is simplest whenever an agent is not part of the group on a trial. The reason for this is that agents which choose independently have access to knowledge of outcomes associated both with the group choice, and with their individual choice, and can directly compare the two. Thus we model the comparative collaboration level outcome on a trial $Z m(t)$ as follows.

$$
Z_{m}(t)=\left\{\begin{array}{lll}
\left(R_{x}(t)+L_{x}(t)\right)-\left(R_{g}(t)+L_{g}(t)\right) & m=1 & 437 \\
\left(R_{g}(t)+L_{g}(t)\right)-\left(R_{x}(t)+L_{x}(t)\right) & m=2 & 438
\end{array}\right.
$$

Here, $R_{x}(t)$ and $L_{x}(t)$ represent rewards and losses on a trial 440 resulting from the individual agent's own card choice $x . R_{g}(t) 441$ and $L_{g}(t)$ represent rewards and losses resulting from the 442 group choice, or vote $g$. The index $m$ indicates whether the 443 comparative value is calculated for the decision to choose 444 independently $m=1$, or the decision to join the collaboration 445 $m=2$.

From here we apply the PVL-Delta model straightforwardly 447 to the decision to collaborate. First, objective value $Z$ is 448 transformed to subjective utility using the prospect utility 449 function. Next, the delta learning rule is applied to update 450 expected value for the two choice options (i.e. join the group 451 or not). Finally, the softmax rule is applied to transform 452 expected values into choice probabilities.

The model for the interaction is necessarily more compli- 454 cated if the agent is part of the collaboration. The reason 455 is that collaborating agents do not naturally have access to 456 knowledge of the outcomes of their own individual choices. 457 For instance, if an agent chooses deck A on a trial, but the 458 group it is collaborating with votes for deck B, then the agent 459 will have information about the outcome associated with the 460 group choice - i.e. the rewards or losses associated with deck 461 $\mathrm{B}$; which it will not have for its own foregone choice - i.e. the 462 rewards or losses associated with deck A.

From here we assume that agents update their evaluations 464 counterfactually on the outcome they believe they would have 465 received, had their choice been realized. In other words, con- 466 sistent with the overall objectives of the study, we assume 467 that collaborating agents compare the experienced value of 468 the group vote, of which they have direct knowledge, with the 469 expected value of their own choice, which they must indirectly 470 infer.

To implement this, we update the expected value of the 472 decision to collaborate as follows.

$$
E v_{m}(t)= \begin{cases}E u_{k}(t-1)-G u_{k}(t) & m=1 \\ G u_{k}(t)-E u_{k}(t-1) & m=2\end{cases}
$$

Here $E v_{m}(t)$ represents the expected value of the collaboration 478 outcomes $m=1$ (choose individually) and $m=2$ (collaborate) 479 on trial $t ; G u_{k}(t)$ represents the outcomes for card choices 480 associated with the group vote; and $E u_{k}(t-1)$ represents the 481 agents own expected value of the card choices, carried over 482 from the previous trial.

To implement confidence sharing in simulations two and 484 three, we assume that in the IGT, agents' confidence is a 485 subjective measure of their relative expectancies for different 486 decision outcomes. Our reasoning here is that an agent who 487 has a much higher expected value for deck A than for decks 488 B, C, and D, will be much more confident in the choice of 489 A, than an agent with a lower expected value for A. Thus, 490 we model confidence sharing within the group as a process of 491 aggregating expectancies across collaborating group members, 492 with the group's choice defined as the alternative with the 493 highest pooled expectancy.

Despite the relative complexity of the interactive scenarios 495 in the simulations, agents are defined simply by the four free 496 
497 parameters in the PVL-Delta model. Recapping, these are 498 the risk preference parameter $A$, loss aversion parameter $w$, 499 learning rate parameter $a$, and consistency parameter $c$; with 500 parameters for both the choice of deck in the IGT, and the 501 decision to collaborate or not.

502 For our simulations to have real world significance and 503 applicability - and for this research to build soundly on the 504 existing empirical literature - it is essential that we do more 505 than implement the best cognitive model available. Empir506 ically appropriate parameter values must also be chosen for 507 that model. Equally important, variability in parameter values 508 between agents must be incorporated in the simulation in a 509 way that reflects variability between human decision makers 510 in the real world. Statistically speaking, this means that the 511 values chosen in our simulations should reflect the distribution 512 of values measured experimentally.

513 To meet this aim, we sample agents' decision parameters

514 from distributions resembling the posterior distributions in515 ferred from experimental data in (29). Specific distributions 516 are shown in Figure 1.

517

518

519

520

521

522

523

524

525

526

527

528

529

530

531

532

533

534

535

536

537

538

539

540

541

542

543

544

545

546

557 being preceded by the decision to collaborate or not. To ensure 558 robustness, each simulation is iterated 100 times.
For each simulation, we report 1) the percentage of agents within a group who commit to the collaboration over trials, 2) the distributions of outcomes (i.e. rewards and losses) on each trial for agents who collaborate, compared to distributions for agents who do not, 3) the cumulative gain/loss of agents throughout each simulation, and 4) the average proportion of choices for each of the decks over time.

Having defined agents' decision parameters at the individual level, we also use Bayesian inference to estimate the same PVL-Delta parameters from the decisions made at the group level. This allows for deeper and more systematic interpretations of the agent-level causes of different patterns of learning and decision making between different group scenarios, thus supporting a more principled linking of micro- and macrolevel level analyses of individual and group choice. By inferring group-level choice parameters in the different simulation scenarios, and relating these to individual agent parameters, we are able to provide more theoretically well-defined predictions for how the different structural features investigated in the simulations might modulate the expression of cognitive characteristics of individual decision makers, and to pinpoint which decision-making processes are the likely causes of differences in group behavior between scenarios.

\section{Simulation Results and Discussion}

Our specific aim in the first simulation is to model the role of knowledge of counterfactual choice alternatives. In this simulation, no confidence sharing takes place, and the group decision is made as a blind vote. In the first asymmetrical knowledge condition, collaborating agents are only given feedback about the outcome of the group decision. Agents in this condition are left to infer what outcome their individual decision might have led to, had they not been part of the group vote. In the second symmetrical knowledge condition, agents are explicitly given counterfactual feedback about the outcome of their individual choice, even if it conflicts with the group.

Figure 2 represents the results of this simulation. The top row of the figure shows that over time, agents in the symmetrical knowledge condition (red) collaborated at a higher rate than agents who received no counterfactual feedback (blue). The second row shows that payoffs were more variable for collaborating agents (lighter colors) in both knowledge conditions, and that agents in the asymmetrical condition earned slightly more if they collaborated, even though they collaborated at a lower rate (light blue). The third row shows that this resulted in a cumulative benefit, with agents in the asymmetrical knowledge condition gaining over the course of the simulation (blue), and agents in the symmetrical condition losing (red). This benefit may be related to differences in learning about risk over time. The bottom row shows that agents in the asymmetrical condition (blue) more quickly learned to avoid the risky Bad deck B, with collaborating agents in this condition (light blue) learning this most quickly. The possible causes of this will be explored below.

Our specific aim in the second simulation is to model the role of more detailed choice integration in the group - in the form of confidence or expectancy sharing - specifically in the asymmetrical knowledge condition (i.e. in the absence of counterfactual knowledge of preferred individual choice alternatives).

Figure 3 represents the results of the asymmetrical knowl- 

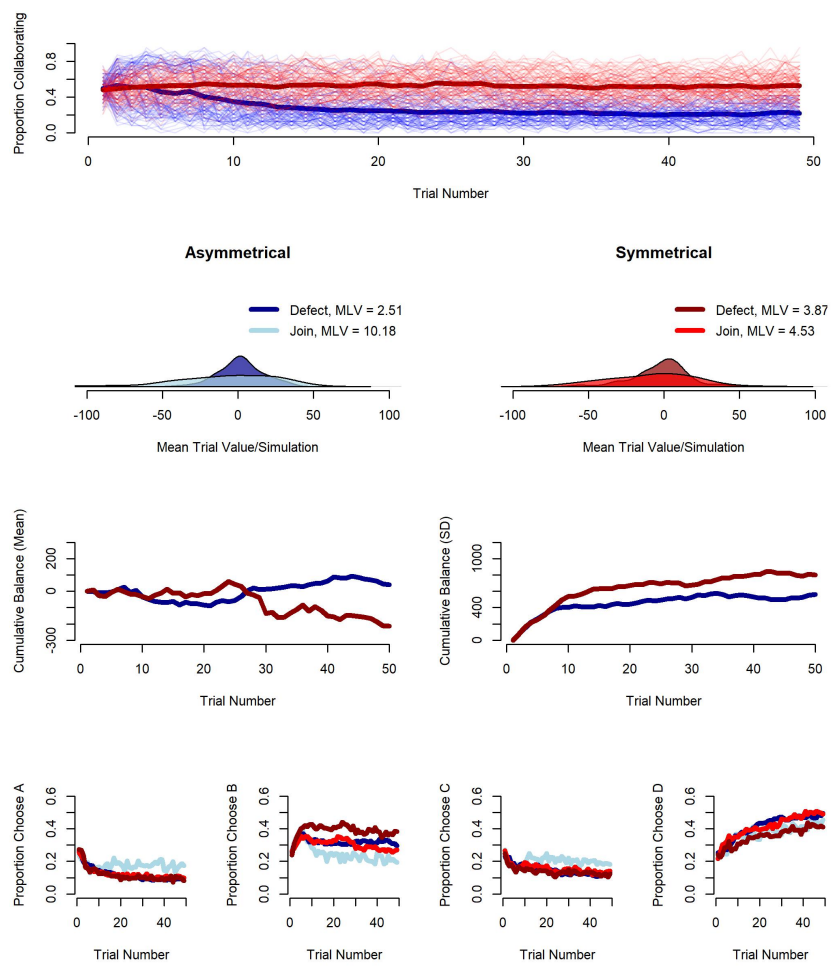

Fig. 2. Theoretical Results Comparing Asymmetrical and Symmetrical Knowledge Conditions. The top row shows the proportion of agents collaborating in the group decision over time. The blue lines represent the proportions for the asymmetrical knowledge condition, and the red lines represent the proportions for the symmetrical condition. Lighter lines represent variability by representing proportions for each iteration of the simulation, and darker lines represent means across all 100 iterations. The second row shows the expected distributions for the value agents receive per trial throughout the simulations. The figures distinguish between the two conditions (hue) and whether or not the agents were part of the collaboration (transparency). The legend displays the mode or most likely value of the distribution. The third row shows the mean (left panel) and standard deviation (right panel) of agents' accumulated gain/loss over time across trials, with the blue and red lines representing asymmetrical and symmetrical knowledge respectively. The bottom row shows the proportion of simulations in which agents in the group (lighter colors), and agents deciding alone (darker colors), chose each of the four decks across the trial runs.

edge condition in simulation 1 , with a new simulation in which the vote was replaced with confidence sharing as described above. The top row shows that confidence sharing has little effect on the rate of collaboration. The second row shows that for agents joining the group (lighter colors), confidence sharing (red) is more likely than voting (blue) to increase payoffs. This is not the case for trials in which agents abstain from the group, suggesting that collective benefit is specifically tied to the group activity. Combined with the lower collaboration rate, this results in the cumulative benefits in both conditions being approximately equal (third row).

Interestingly, the bottom row of the figure suggests that the collective benefit for confidence sharing may be more a result of appropriate choice parameters at the group level, and less a result of the group context facilitating learning about the IGT payoff environment. Agents who join the group in the confidence sharing simulation almost immediately show low preferences for the risky and disadvantageous deck B, and high preferences for the favorable deck D.

This interpretation can be explored more fully by investi-
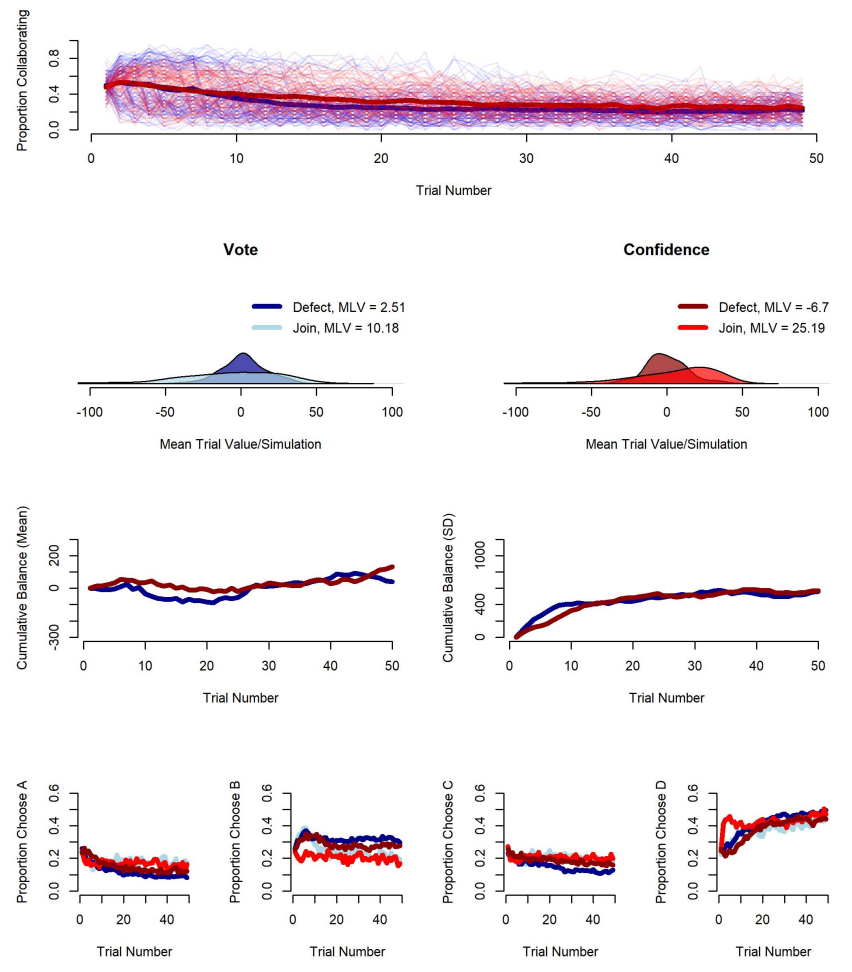

704

705

706

707

708

Fig. 3. Theoretical Results Comparing Voting and Confidence Sharing Under Asymmetrical Knowledge Conditions. The top row shows the proportion of agents collaborating in the group decision over time. The blue lines represent the proportions for the voting agents, and the red lines represent the proportions for the confidence sharing agents. The second row shows the expected distributions for the value agents receive per trial throughout the simulations. The legend displays the mode or most likely value of the distribution. The third row shows the mean and standard deviation of agents' accumulated gain/loss over time across trials, with the blue and red lines representing voting and confidence sharing respectively. The bottom row shows the proportion of simulations in which agents in the group, and agents deciding alone, chose each of the four decks across the trial runs.

gating the inferred group-level PVL-Delta model parameters, 722 posterior distributions for which are shown in Figure 4. This 723 figure shows that in the asymmetric knowledge condition, the 724 group context has little effect on the consistency parameter 725 c. More interestingly, the figure shows that the confidence 726 sharing group (red) has much lower estimates for the learning 727 rate parameter $a$, with learning rate converging on zero. The 728 figure also shows that the estimated learning rate is increased 729 for the voting group.

In contrast, the figure shows that confidence sharing in- 731 creased the risk preference parameter $A$, and increased the 732 loss aversion parameter $w$. Interpreted within prospect theory, 733 the increased $A$ parameter suggests that confidence sharing 734 promotes a more linear value function. Theoretically, this 735 corresponds to a decreased sensitivity to risk, in the form of 736 decreased risk aversion for gains, and decreased risk seeking 737 for losses. The increased $w$ parameter suggests that confidence 738 sharing increases group loss aversion (i.e. the influence of 739 losses on expectancies, compared to gains).

Taken together, these differences support an interpretation 741 that, in the absence of knowledge of forgone individual out- 742 comes (i.e. when knowledge of group and individual outcomes 743 is asymmetrical), voting and confidence sharing both enhance 744 

805 at which it chooses the Good deck D.

806 This interpretation is supported by investigation of the
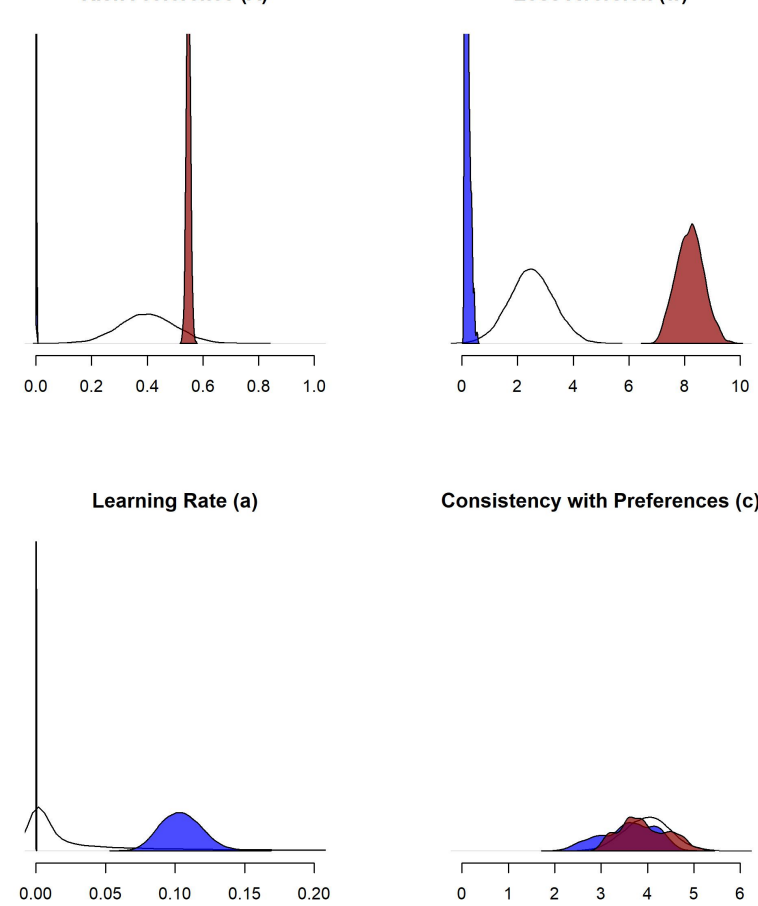

Fig. 4. Posterior Parameter Estimates from Application of the Individual PVL-Delta Model to Group-Level Choices In Voting and Confidence Sharing Groups In the Asymmetric Knowledge Condition. Blue distributions represent posteriors for voting, red distributions represent posteriors for confidence sharing.

cumulative gain over time, and that interestingly, they do so by different mechanisms. Voting promotes group learning, while confidence sharing suppresses group learning but promotes generally more appropriate decision parameters for balancing risk and utility from the outset.

Our specific aim in the third simulation is to model the same group processes in the symmetrical knowledge condition, in which agents do received counterfactual knowledge of the outcomes they would have received had they not committed to the group. Figure 5 represents the results of this simulation. The figure presents the outcome of the symmetrical knowledge condition in simulation 1 , with a new simulation in which the vote was replaced with confidence sharing.

The top row of the figure shows that counterfactual knowledge of individually preferred choice options increases rate of collaboration for both kinds of groups. The second row of the figure shows that in this condition, committing to the confidence sharing group leads to collective gain, however this gain is not as large or as clear as it is in the asymmetrical knowledge condition. The third row shows that with agents collaborating more frequently, there is an increased cumulative ain over trials.

In contrast to the asymmetrical knowledge condition, this cumulative gain appears to be linked to improved learning for gents in the confidence sharing condition. The bottom row hows that the confidence sharing group decreases the rate at
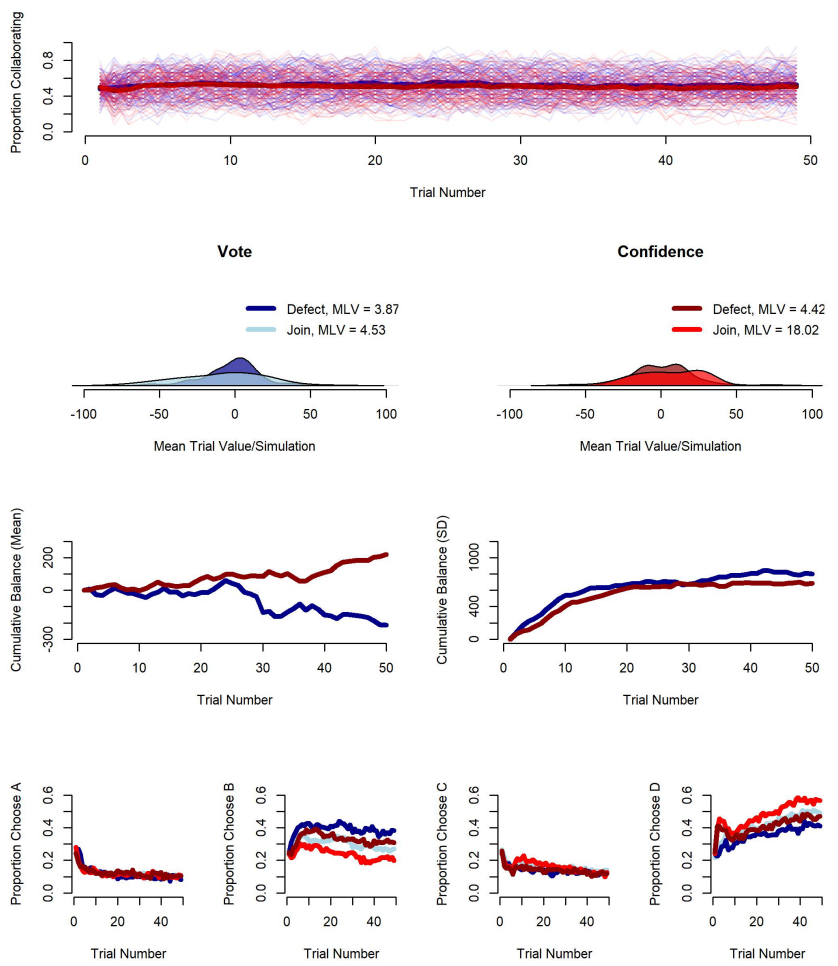

828

Fig. 5. Theoretical Results Comparing Voting and Confidence Sharing Under Symmetrical Knowledge Conditions. The top panel shows the percentage of agents collaborating in the group decision over time. The second row shows expected value distributions, and the third row shows cumulative payoffs over time. THe bottom row represents choice preferences over trials in each group and condition. Coloring and lightness represent group collaboration and the voting and confidence sharing conditions as in Figures 2 and 3.

group-level PVL-Delta parameters, shown in Figure 6. The confidence sharing group has an increased learning rate relative to the voting group. However, it should be noted that this rate is less than the learning rate for the voting group in the symmetrical voting condition. Moreover, the figure also shows that, as for the asymmetrical knowledge condition, the confidence sharing group has a more appropriate Prospect Theoretic loss aversion parameter. Thus, cumulative gain associated with confidence sharing when knowledge of forgone individual outcomes $i s$ available, seems to result from a combination of learning and more appropriate choice parameters.

\section{Summary and Conclusion}

Together these three simulations suggest the following empirical predictions. When autonomous agents are required to make risky decisions in open collaborative groups, they will commit to the group more readily if they are given knowledge of how they would have fared, had they made their decisions alone; even if they would have fared better. This may occur as a result of realistic counterfactual feedback tempering overly optimistic expectancies for preferred choice alternatives. With such feedback, the group may provide a valuable regulatory function in learning about the value of collaborative decision making.

The simulations also supports a growing body of research
834

835

836 


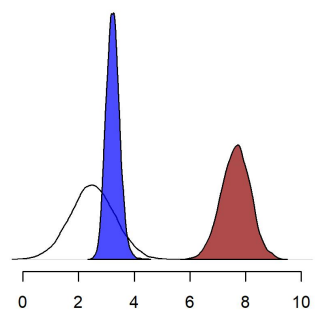

Consistency with Preferences (c)

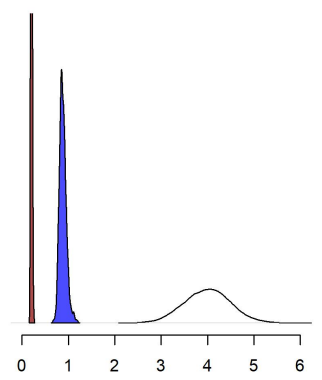

Fig. 6. Posterior Parameter Estimates from Application of the Individual PVL-Delta Model to Group-Level Choices In Voting and Confidence Sharing Groups In the Asymmetric Knowledge Condition. Blue distributions represent posteriors for voting, red distributions represent posteriors for confidence sharing.

showing that confidence sharing generally leads to increased decision performance. Importantly, the simulations suggest interesting interactions between confidence sharing, voting, and knowledge of forgone individual outcomes. When such knowledge is not provided, and groups are collaborating at a relatively low rate, group benefits will occur via different mechanisms, depending on the method of choice aggregation. Groups which decide by vote will gain collective benefit over time via improved learning. In contrast, groups which decide by confidence sharing will gain collective benefit by virtue of group dynamics leading to decision parameters which are better suited to risk and loss in the environment.

The same is not the case when knowledge of forgone individual outcomes is provided. Here, groups which decide by vote will collaborate more, but we should not expect them to derive a collective benefit. In contrast, groups which decide by confidence sharing will both collaborate more and derive a collective benefit. Moreover, this collective benefit will be produced by a combination of increased group learning, and more suitable decision parameters for balancing risk and loss.

Of course not all organizational forms are conducive to dispersed and emergent organizing. Nevertheless, for those institutional forms that rely on autonomous individuals with latent and often non-utilized knowledge, our results indicate that these individuals' knowledge can be harnessed in powerful ways, and that decision making teams can be more stable, and their individuals more successful, if simple design principles such as counterfactual knowledge and confidence sharing are implemented mindfully within the group. This suggests that 931 decentralized organizational forms function best when they 932 are not only structurally open, but informationally open as 933 well. Such a conclusion provides important directions for 934 future research - not only theoretical, but also experimental 935 and qualitative - on the role of information in decentralized 936 $\begin{array}{ll}\text { decision making groups. } & 937\end{array}$

ACKNOWLEDGMENTS. Thanks to Michael Lee for helpful com- 939 ments on an early draft. practical reasoning in

2. Kollman K, Miller JH, Page SE (1998) Political parties and electoral landscapes. British Jour- 943 nal of Political Science 28(1):139-158. 944 3. Kollman K, Miller JH, Page SE (2003) Computational Models in Political Economy. (MIT 945
Press).

4. Harhoff D, Lakhani KR (2016) Revolutionizing Innovation: Users, Communities, and Open 946 $\begin{array}{ll}\text { Innovation. (MIT Press). } & 947\end{array}$

5. Felin T, Lakhani KR, Tushman ML (2017) Firms, crowds, and innovation. Strategic Organiza- 948

. Brafman O, Beckstrom R (2006) The Starfish and the Spider: The Unstoppable Power of 949

$\begin{array}{ll}\text { Leaderless Organizations. (Penguin). } & 950\end{array}$

7. von Hippel E (2005) Democratizing Innovation. (MIT Press).

8. Puranam P, Håkonnson D (2015) Valve's way. Journal of Organizational Design 4(2):3-4.
9. Foss NJ (2003) Selective intervention and internal hybrids: Interpreting and learning from the 952 $\begin{array}{ll}\text { rise and decline of the oticon spaghetti organization. Organization Science 14(3):331-349. } & 953\end{array}$

10. Grosskopf B, Erev I, Yechiam E (2006) Forgone with the wind: Indirect payoff information and 953 its implications for choice. International Journal of Game Theory 34:285-302.

11. Rakow T, Newell B, Wright $L$ (2015) Forgone but not forgotten: The effects of partial and full 955 feedback in "harsh" and "kind" environments. Psychonomic Bulletin and Review 22(6):1807- 956
1813.

12. Yechiam E, Rakow $T$ (2012) The effect of forgone outcomes on choices from experience: An 957 individual level modeling analysis. Experimental Psychology 59:55-67. 958

13. Tsiros M, Mittal V (2000) Regret: A model of its antecedents and consequences in consumer 959 decision making. Journal of Consumer Research 26(4):401-417.

14. Zeelenberg M, Beattie J, van der Pligt J, de Vries NK (1996) Consequences of regret aver- 960 sion: Effects of expected feedback on risky decision making. Human Decision Processes 961 65(2):148-158.

15. Thomas JP, McFadyen RG (1995) The confidence heuristic: A game-theoretic analysis. Jour- 962 nal of Economic Psychology 16(1):97-113.

16. Bahrami B, et al. (2010) Optimally interacting minds. Science 7:1081-1085.

17. Fusaroli R, et al. (2012) Coming to terms: quantifying the benefits of linguistic coordination 964 Psychological Science 23:931-939. 965

18. Mahmoodi A, et al. (2015) Equality bias impairs collective decision-making across cultures. 966 Proceedings of the National Academy of Sciences 112(12):3835-3840.

19. Mahmoodi A, Bang D, Ahmadabadi MN, Bahrami B (2013) Learning to make collective deci- 967 sions: The impact of confidence escalation. PLoS One 8(12). 968

20. Bang D, et al. (2017) Confidence matching in group decision-making. Nature Human Be- 969

21. Hahn RW, Tetlock PC (2006) Information Markets: A New Way of Making Decisions. (AEI 970 Brookings).

2. Rhode PW, Strumf KS (2004) Historic presidential betting markets. Journal of Economic

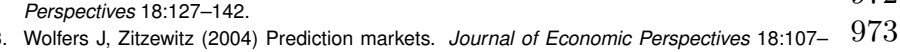
126.

24. Hueffer K, Fonseca MA, Leiserowitz A, Taylor KM (2013) The wisdom of crowds: Predicting 975 a weather and climate related event. Judgment and Decision Making 8(2):91-105.

25. Bechara A, Damásio AR, Damásio H, Anderson SW (1994) Insensitivity to future conse- 976 quences following damage to human prefrontal cortex. Cognition 50(1-3):7-15. 977

26. Bechara A, H. D, Tranel D, Damasio AR (1997) Deciding advantageously before knowing the 978 advantageous strategy. Science 275(5304):1293-1295.

27. March JG (1991) Exploration and exploitation in organizational learning. Organization Sci- 979 ence 2(1):71-87.

28. Ahn WY, Busemeyer JR, Wagenmakers EJ, Stout JC (2008) Comparison of decision learning 980 models using the generalization criterion method. Cognitive Science 32:1376-1402.

29. Steingroever H, Wetzels R, Wagenmakers EJ (2013) validating the pvl-delta model for the 982 iowa gambling task. Frontiers in Psychology 4(898).

30. Steingroever $\mathrm{H}$, Wetzels R, Wagenmakers EJ (2013) A comparison of reinforcement-learning models for the iowa gambling task using parameter space partitioning. Journal of Problem Solving $5(2)$.

31. Busemeyer JR, Stout JC (2002) A contribution of cognitive decision models to clinical assessment: Decomposing performance on the bechara gambling task. Psychological Assessment 14(3):253-262.

32. Kahneman D, Tversky A (1979) Prospect theory: An analysis of decision under risk. Econometrica 47(2):278-288.

33. Halberstadt J, et al. (2016) Incipient social groups: An analysis via in-vivo behavioural tracking. PLoS One 11(13).

34. Raine L, Purcell K, Smith A (2011) The social side of the internet. 